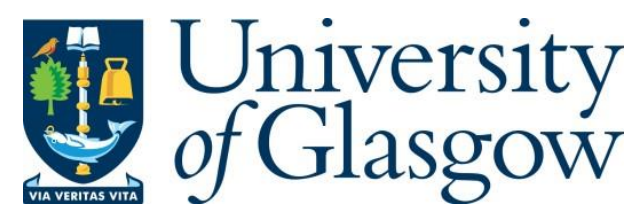

Kang, L., Feeney, A., Somerset, W. and Dixon, S. (2019) Wideband Electromagnetic Dynamic Acoustic Transducer as a Standard Acoustic Source for Air-coupled Ultrasonic Sensors. In: 2019 IEEE International Ultrasonics Symposium (IUS), Glasgow, UK, 0609 Oct 2019, pp. 2481-2484. ISBN 9781728145969.

There may be differences between this version and the published version. You are advised to consult the publisher's version if you wish to cite from it.

http://eprints.gla.ac.uk/215497/

Deposited on: 2 July 2020

Enlighten - Research publications by members of the University of Glasgow http://eprints.gla.ac.uk 


\title{
Wideband Electromagnetic Dynamic Acoustic Transducer as a Standard Acoustic Source for Air-coupled Ultrasonic Sensors
}

\author{
Lei Kang, Andrew Feeney, Steve Dixon \\ University of Warwick, United Kingdom \\ Email: L.Kang.1@warwick.ac.uk; victorkang11@126.com
}

\begin{abstract}
To experimentally study the characteristics of ultrasonic sensors, a wideband air-coupled ultrasonic transducer, wideband electromagnetic dynamic acoustic transducer (WEMDAT), is designed and fabricated. Characterisation methods, including electrical impedance analysis, laser Doppler vibrometry and pressure-field microphone measurement, are used to examine the performance of the WEMDAT, which have shown that the transducer has a wide bandwidth ranging approximately from $47 \mathrm{kHz}$ to $145 \mathrm{kHz}$ and a good directivity with a beam angle of around $20^{\circ}$ with no evident side lobes. A $40 \mathrm{kHz}$ commercial flexural ultrasonic transducer (FUT) is then taken as an example to receive ultrasonic wave in a pitch-catch configuration to evaluate the performance of the WEMDAT as an acoustic source. Experiment results have demonstrated that the WEMDAT can maintain the most of the frequency contents of a 5 cycle $40 \mathrm{kHz}$ tone burst electric signal and convert it into an ultrasonic wave for studying the dynamic characteristic and the directivity pattern of the ultrasonic receiver. A comparison of the dynamic characteristics between the transmitting and the receiving processes of the same FUT reveals that the FUT has a wider bandwidth when operating as an ultrasonic receiver than operating as a transmitter, which indicates that it is necessary to quantitatively investigate the receiving process of an ultrasonic transducer, demonstrating a huge potential of the WEMDAT serving as a standard acoustic source for ultrasonic sensors for various air-coupled ultrasonic applications.
\end{abstract}

Keywords - wideband electromagnetic dynamic acoustic transducer, air-coupled ultrasounic measurement, acoustic source, flexural ultrasonic transducer, characterisation.

\section{INTRODUCTION}

Air-coupled ultrasonic transducers, such as flexural ultrasonic transducers (FUT) and piezoelectric-based ultrasonic transducers with matching layer(s), have been broadly utilised in various ultrasonic applications including distance ranging, proximity sensing, flow measurement and non-destructive testing and evaluation ${ }^{[1-10]}$. Understanding the characteristics and performance of the ultrasonic transducers is essential when high-accuracy measurement is highly demanded. The transmitting process of an ultrasonic transmitter has been extensively investigated both theoretically and experimentally ${ }^{[2-10]}$, and many devices and instruments, such as laser Doppler vibrometers, microphones, and hydrophones, have been designed for qualitatively investigating the dynamic characteristics of fluid-coupled ultrasonic transmitters. However, the means for experimentally studying the characteristics of ultrasonic sensors are relatively limited.

A novel type of wideband air-coupled ultrasonic transducers, wideband electromagnetic dynamic acoustic transducer (WEMDAT), has been recently developed [2]. Initial measurement results have demonstrated that it has good performance in terms of transduction efficiency, bandwidth and radiation pattern and it is able to operate as either an air-coupled ultrasonic transmitter or a receiver. To further explore the potential of this type of transducer, this paper investigates its characteristics and applications serving as a standard acoustic source.

\section{DESGIN AND CHARACTERISATION OF A WEMDAT}

The basic structure of a WEMDAT consists a ring magnet, a solenoid with a magnetic core and a non-magnetic conductive foil. The edge of the membrane is fixed on the top of the magnet, in the vicinity of the solenoid. The solenoid is fixed to the threaded magnetic core. When an alternating current is fed into the solenoid, an eddy current will be induced in the foil; the moving electrons will experience a Lorentz force in a static magnetic field, and thus collide the atoms of the foil, resulting in a vibration of the foil. The foil thus serves as an acoustic source, generating ultrasonic waves in fluids such as air.

A WEMDAT prototype has been designed, whose assembly schematic is shown in Fig. $1^{[2]}$. To function as a standard acoustic source for ultrasonic sensors or receivers, the WEMDAT should have a wideband frequency response, so that it is feasible for ultrasonic sensors operating at different frequencies. Both of the electrical and the mechanical sections of a WEMDAT influence the overall bandwidth performance of the transducer. To broaden its bandwidth, a low inductance magnetic core such as steel is utilised to reduce the quality factor of the electrical characteristics of the transducer. Moreover, thin foil such as a $15 \mu \mathrm{m}$ thick aluminium foil is chosen to avoid significant mechanical resonant vibration during the operation of the WEMDAT. In addition to the bandwidth performance, the transduction efficiency of a WEMDAT should also be considered. Several approaches have 
been adopted for enhancing the transduction efficiency of the WEMDAT. The distance of between the solenoid and the foil is designed to be adjustable so that the smallest distance can be achieved for different levels of driving power; a strong rareearth magnet such as a neodymium iron boron magnet is utilised to enhance the magnetic field in the foil; and, a light and thin foil is also helpful for the enhancement of the efficiency.

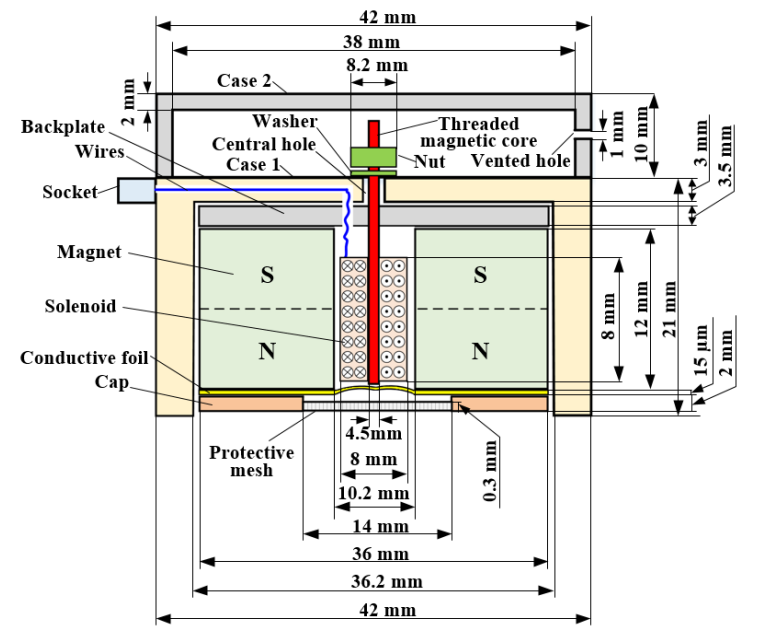

Fig. 1. Assembly schematic of a WEMDAT prototype (patent applied for).

A WEMDAT has been fabricated and assembled according to Fig. 1. Its electrical and acoustic characteristics are further investigated through impedance analysis, laser Doppler vibrometer and wideband microphone.

The electrical characteristics of the WEMDAT is studied by an electrical impedance analyser (Agilent 4294A Precision Impedance Analyser). The impedance and the phase of the WEMDAT are shown in Fig. 2, which demonstrates that there is no significant electrical resonance in the transducer. To further evaluate the overall bandwidth of the WEMDAT, the transducer is configured as a transmitter, operating with a calibrated wideband microphone (B\&K 4138-A-015), in a pitch-catch mode with a separation of around $206 \mathrm{~mm}$. The WEMDAT is driven by a wideband signal with a peak-to-peak voltage of $7.1 \mathrm{~V}$, directly output by a function generator (Tektronix AFG3102C). The frequency spectra of the driving voltage signal and the sound pressure signal detected by the microphone are shown in Fig. 3. As the effective frequency range of the microphone is below $120 \mathrm{kHz}$, the frequency spectrum of the driving signal is set to the range from 0 to 200 $\mathrm{kHz}$. The WEMDAT exhibits a broadband characteristic, with a $-6 \mathrm{~dB}$ bandwidth ranging from $47 \mathrm{kHz}$ to $145 \mathrm{kHz}$.

The amplitude of the displacement of the foil of the WEMDAT is then scanned point by point utilising a laser Doppler vibrometer (Polytec OFV-5000), which indicates that an annular-like acoustic source is formed by the WEMDAT, as shown in Fig. 4. The resultant radiation pattern of the acoustic source is then measured utilising the microphone, by rotating the WEMDAT from $-90^{\circ}$ to $90^{\circ}$ with a step of $2^{\circ}$. The radiation pattern is shown in Fig. 5, which indicates that the WEMDAT has a good directivity with a $-3 \mathrm{~dB}$ beam angle of around $20^{\circ}$ and no evident side lobes exist in the radiation pattern.

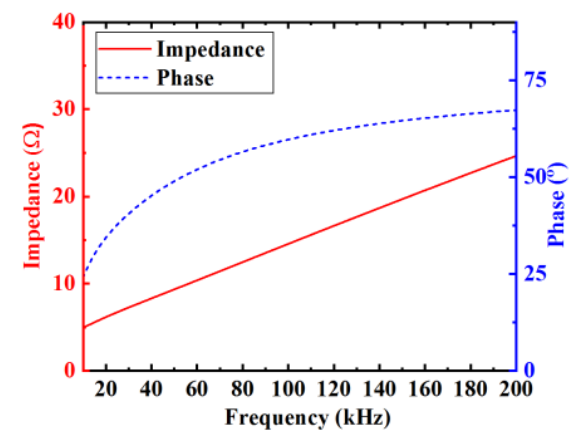

Fig. 2. Impedance and phase of the WEMDAT in the range of 0 to $200 \mathrm{kHz}$

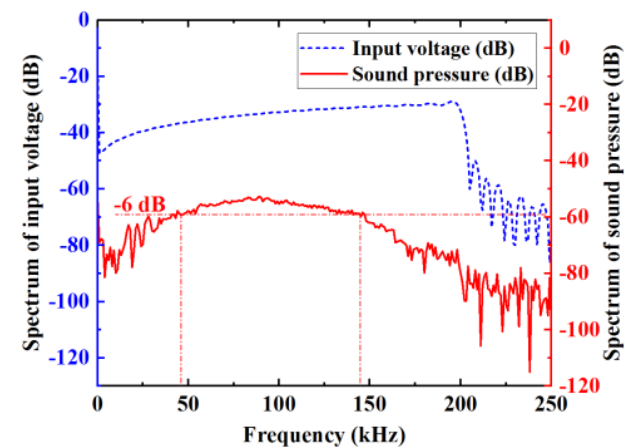

Fig. 3. The spectra of the input voltage signal on the WEMDAT and the spectrum of the sound pressure signal received by a calibrated wideband microphone, which demonstrates the wideband performance of the WEMDAT.

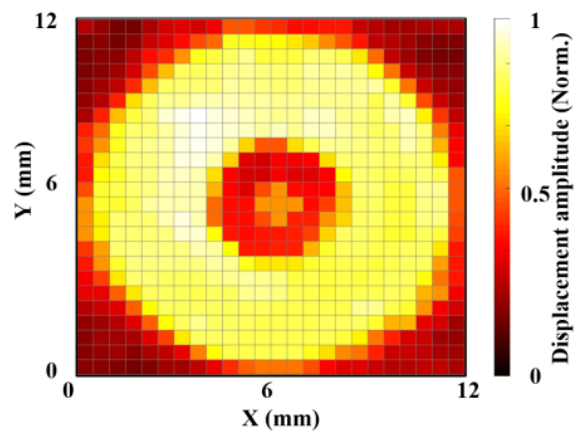

Fig. 4. The displacement amplitude of the membrane of the WEMDAT driven by a sinc function signal with a frequency spectrum ranging from 0 to $200 \mathrm{kHz}$, showing that an annular-like acoustic source is formed by the WEMDAT.

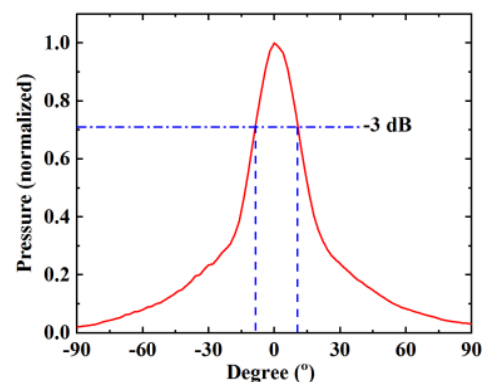

Fig. 5. The radiation pattern of the WEMDAT measured by the calibrated microphone, showing that the WEMDAT has a good directivity with a $-3 \mathrm{~dB}$ full beam angle of approximately $20^{\circ}$.

\section{EXPERIMENTS}

To evaluate the performance of the WEMDAT as a standard acoustic source, the microphone is firstly chosen as a receiver, 
operating with the WEMDAT in a pitch-catch configuration with a separation of approximately $271 \mathrm{~mm}$. A $40 \mathrm{kHz} 5$-cycle tone burst voltage signal directly output by the function generator is taken as an example to drive the WEMDAT. The driving signal and the sound pressure signal and their spectra are shown in Fig. 6. This figure demonstrates that, the WEMDAT can effectively working as a wideband acoustic source, converting the transmitting voltage signal into an ultrasonic wave, and meanwhile, maintaining the majority of the frequency contents of the voltage signal.

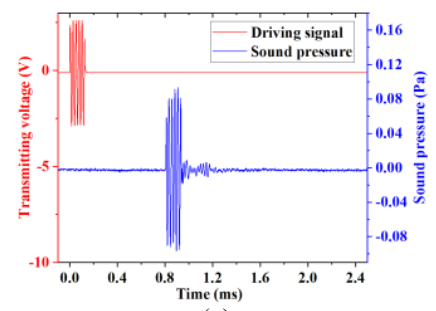

(a)

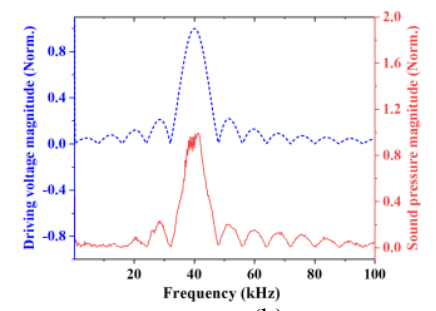

(b)
Fig. 6. The WEMDAT is configured as an ultrasonic transmitter and a calibrated wideband microphone is configured as an ultrasonic receiver, with a separation of around $271 \mathrm{~mm}$, showing (a) The transmitting voltage signal on the WEMDAT and the sound pressure signal detected by the microphone in temporal domain, and (b) The spectra of the transmitting signal and the pressure signal in frequency domain.

A $40 \mathrm{kHz}$ flexural ultrasonic transducers (Multicomp), FUT1, is then configured as a receiver in the same experimental set-up, where the WEMDAT serves as a transmitter. The received signal is amplified by a low-noise wideband amplifier (SAA1000, Sonemat) with a fixed gain of approximately 35 . The driving and the receiving voltage signals and their frequency spectra are shown in Fig. 7. As the nominal bandwidth of FUT1 is around $1 \mathrm{kHz}$, the $-3 \mathrm{~dB}$ bandwidth of the received signal is significantly reduced to around $2.51 \mathrm{kHz}$. The decrease in the bandwidth results in a longer time duration in time domain, as shown in Fig. 7 (a). In pitch-catch and pulseecho ultrasonic measurement configurations, understanding the dynamic characteristic of the transmitter and the receiver is essential for mathematically modelling a complete ultrasonic transmitting, propagating and receiving process. Microphone and laser Doppler vibrometer have been widely utilised to investigate the transmitting process. Fig. 7 demonstrates that, with the WEMDAT, it is possible to conveniently study the dynamic characteristics in the receiving process of an ultrasonic sensor.
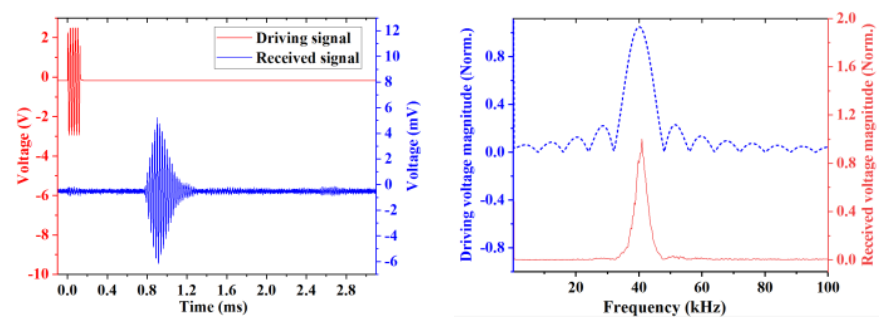

Fig. 7. The WEMDAT is configured as an ultrasonic transmitter and FUT1 is configured as an ultrasonic receiver, with a separation of around $271 \mathrm{~mm}$, showing (a) The transmitting voltage signal on the WEMDAT and the received voltage signal detected by FUT1 in temporal domain, and (b) The spectra of the transmitting and the receiving signals in frequency domain.
For an ultrasonic transducer which operates as both a transmitter and a receiver, its dynamic characteristic in the transmitting process can be different from that in the receiving process, which is illustrated in Fig. 8, where FUT1 is configured as the transmitter and the microphone serves as the receiver. The received signal has longer duration in temporal domain, and its $-3 \mathrm{~dB}$ bandwidth is around $1.76 \mathrm{kHz}$. This is even narrower than the spectrum shown Fig. 7 (b) where FUT1 serves as a receiver and the WEMDAT is configured as the transmitter. According to the datasheet of the microphone, its frequency response characteristic in the range from $20 \mathrm{~Hz}$ to $100 \mathrm{kHz}$ is much flatter than that of the WEMDAT, the only reason for the bandwidth shown in Fig. 8 (b) is narrower than that shown in Fig. 7 (b) seems to be that the FUT1 has a narrower bandwidth when operating as a transmitter than operating as a receiver. This proves that, assuming the dynamic characteristic in the transmitting process of the transducer is the same as in the receiving process can be problematic in establishing a complete mathematical model, or, in the accurate time-of-flight measurement of ultrasonic wave where the time delays in both the transmitting and the receiving transducers should be considered.

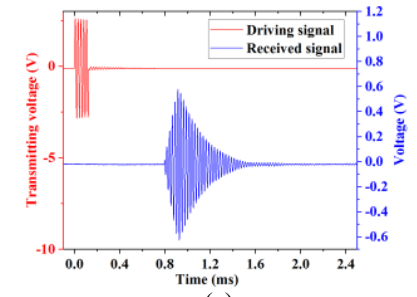

(a)

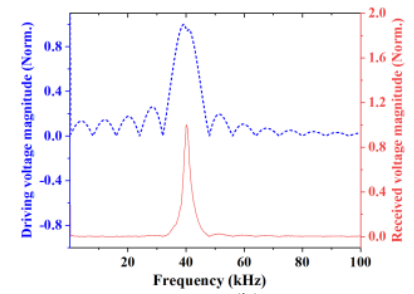

(b)
Fig. 8. A $40 \mathrm{kHz}$ commercial FUT (FUT1) is configured as an ultrasonic transmitter and a calibrated wideband microphone is configured as an ultrasonic receiver, with a separation of around $271 \mathrm{~mm}$, showing (a) The transmitting voltage signal on the FUT1 and the sound pressure signal detected by the microphone in temporal domain, and (b) The spectra of the transmitting signal and the pressure signal in frequency domain.

Using the same experimental set-up, the dynamic characteristics of two $40 \mathrm{kHz}$ commercial FUTs are further investigated, where FUT1 is configured as a receiver and FUT2 is configured as a transmitter. The transmitted voltage and the received voltage signals are shown in Fig. 9 (a) and their spectra are shown in Fig. 9 (b). As both of the FUTs are narrow-band devices whose nominal bandwidth is around $1 \mathrm{kHz}$, the received ultrasonic signal has an even longer time duration whose corresponding $-3 \mathrm{~dB}$ bandwidth is only $0.99 \mathrm{kHz}$. A comparison between Figs. 7 and 9 shows that the amplitude of the ultrasonic signal is around 7.7 times greater in Fig.9, at a price of a longer time duration and less steep envelope. In ultrasonic measurement applications where the measurement accuracy of the time-of-flight is highly demanded, a short temporal signal with a steep envelope is advantageous for increasing the measurement accuracy. A combination of a WEMDAT with a narrowband ultrasonic receiver can significantly shorten the duration and sharpen the envelope of the signal, so that a compromise between signal's amplitude and signal's bandwidth can be achieved. 


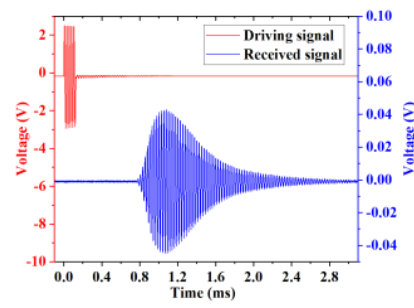

(a)

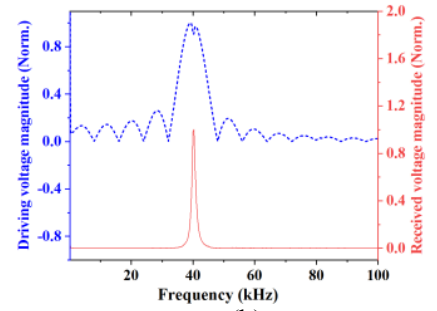

(b)
Fig. 9. Two $40 \mathrm{kHz}$ commercial FUTs, FUT2 and FUT1, are respectively configured as an ultrasonic transmitter and a an ultrasonic receiver, with a separation of around $271 \mathrm{~mm}$, showing (a) The transmitting voltage signal on the transmitting FUT2 and the received voltage signal detected by the receiving FUT1 in temporal domain, and (b) The spectra of the transmitting and the receiving signals in frequency domain.

In addition to studying the dynamic process of the ultrasonic sensors, the WEMDAT is also utilised to measure the directivity pattern of the FUT1. Similar to the measurement of a radiation pattern of an ultrasonic transmitter using a microphone, the WEMDAT is rotated from $-90^{\circ}$ to $90^{\circ}$ with a step of $2^{\circ}$ whilst the position of FUT1 is fixed. The directivity pattern of FUT1 operating as an ultrasonic receiver is shown in Fig. 10, where the $-3 \mathrm{~dB}$ beam angle is around $51.4^{\circ}$.

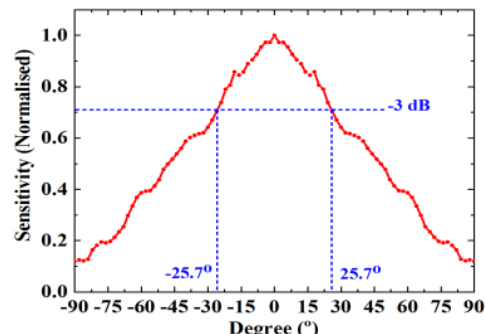

Fig. 10. The directivity pattern of FUT1 operating as an ultrasonic receiver, utilising the WEMDAT as an acoustic source, showing that the FUT has a $-3 \mathrm{~dB}$ full beam angle of around $51.4^{\circ}$.

Calibrated acoustic microphones are commonly used to characterise the acoustic radiation pattern of ultrasonic transmitters, but the use of such calibrated acoustic microphones can be prohibitive and provide limited opportunity for performance optimisation for ultrasonic sensors. All experiment results shown above have demonstrated that the WEMDAT prototype can operate effectively as a standard acoustic source for investigating the dynamic characteristics and the directivity pattern of ultrasonic sensors, displaying significant potential as an optimisable standard acoustic source for investigating and characterising ultrasonic sensors.

\section{CONCLUSIONS}

A wideband air-coupled ultrasonic transducer, wideband electromagnetic dynamic acoustic transducer (WEMDAT), is designed, fabricated and characterised. The WEMDAT has wideband performance and a good directivity. It can be directly driven a function generator, converting a 5 cycle $40 \mathrm{kHz}$ tone burst signal into ultrasonic wave and meanwhile maintaining the most contents of the driving signal. The WEMDAT has been utilised to study the receiving dynamic characteristic and the directivity pattern of a $40 \mathrm{kHz}$ flexural ultrasonic transducer
(FUT). It has been found that the FUT has a wider bandwidth when operating as an ultrasonic receiver than operating as a transmitter, which demonstrates a huge potential of the WEMDAT serving as a standard acoustic source for ultrasonic sensors for various air-coupled ultrasonic applications.

\section{ACKNOWLEDGMENT}

The authors would like to acknowledge the European Union's SACUT project (Ref. No. 612118) under the Marie Curie Industry-Academia Pathways \& Partnership (IAPP) action and the EPSRC grant EP/N025393/1, for funding the research.

\section{REFERENCES}

[1] D. E. Chimenti, "Review of air-coupled ultrasonic materials characterization," Ultrasonics, vol. 54, no. 7, pp. 1804-1816, 2014.

[2] L. Kang, A. Feeney, and S. Dixon., "Wideband electromagnetic dynamic acoustic transducers (WEMDATs) for air-coupled ultrasonic applications,” Appl. Phys. Lett., vol. 114, no. 5, p. 053505, Jan. 2019.

[3] L. Kang, T. Eriksson, S.N. Ramadas, et al. "Design of flexural ultrasonic phased array for fluid-coupled applications", 2016 IEEE Ultrasonics Symposium, Tours, France, pp. 1-4, 2016.

[4] L. Kang, A. Feeney, R. Su, et al. "Two-dimensional flexural ultrasonic phased array for flow measurement," 2017 IEEE Ultrasonics Symposium, Washington DC, USA, pp. 1-4, 2017.

[5] T.J.R. Eriksson, S.N. Ramadas, and S. Dixon, "Experimental and simulation characterisation of flexural vibration modes in unimorph ultrasound transducers," Ultrasonics, vol. 65, pp. 242-248, 2016.

[6] A. Feeney, L. Kang, and S. Dixon, "High frequency measurement of ultrasound using flexural ultrasonic transducers," IEEE Sensors J., vol. 18, no. 13, pp. 5238 - 5244, Jul. 2018.

[7] A. Feeney, L. Kang, G. Rowlands, L. Zhou, and S. Dixon, "Dynamic nonlinearity in piezoelectric flexural ultrasonic transducers", IEEE Sensors J., vol. 19, no. 15, pp. 6056-6066, 2019.

[8] S. Dixon, L. Kang, M. Ginestier, C. Wells, G. Rowlands, and A. Feeney, The electro-mechanical behaviour of flexural ultrasonic transducers," Appl. Phys. Lett., vol. 110, no. 22, p. 223502, 2017.

[9] A. Feeney, L. Kang, G. Rowlands, and S. Dixon, "The dynamic performance of flexural ultrasonic transducers," Sensors, vol. 18, no. 1, p. $270,2018$.

[10] A. Feeney, L. Kang, and S. Dixon, "Nonlinearity in the dynamic response of flexural ultrasonic transducers," IEEE Sensors Lett., vol. 2, no. 1, pp. 1-4, Mar. 2018. 